\title{
Antibiotic resistance of bacteria responsible of acute respiratory tract infections in children
}

\author{
Makhtar Camara, ${ }^{1}$ Assane Dieng, ${ }^{1}$ Abdoulaye Diop, ${ }^{1}$ Amadou Diop, ${ }^{1}$ Amadou Diop, ${ }^{2}$ Djibril Boiro, ${ }^{3}$ \\ Jean Baptisse Niokhor Diouf, ${ }^{4}$ Amary Fall, ${ }^{5}$ Ousmane Ndiaye, ${ }^{3}$ Mbayame Niang, ${ }^{5}$ Cheikh Saad Bouh Boye ${ }^{1}$ \\ ${ }^{1}$ Bacteriology and Virology Laboratory, Le Dantec teaching Hospital, Dakar; ${ }^{2}$ Bacteriology and Virology Laboratory, \\ Albert Royer teaching Hospital, Dakar; ${ }^{3}$ Paediatric Unit, Abass NDAO Teaching Hospital, Dakar; ${ }^{4}$ Paediatric Unit, \\ Roi Baudouin Hospital, Dakar; ${ }^{5}$ Medical Virology Unit, Institute Pasteur, Dakar, Senegal
}

\section{Summary}

Background and aims. Streptococcus pneumoniae, Haemophilus influenzae and Moraxella catarrhalis are the most common causative agents of acute respiratory tract infections (RTIs). The objective of this study was to assess their susceptibility to several antibiotics.

Materials and methods. A total of 58 strains (16 S. pneumoniae, $19 \mathrm{H}$. influenzae and $23 \mathrm{M}$. catarrhalis) were isolated from samples collected in two paediatric centres, and their

Correspondence: Makhtar Camara, CHNU Aristide Le Dantec, Cheikh Anta Diop University, Laboratory of Bacteriology-Virology, 30 Avenue Pasteur, BP 7325, Dakar, Senegal.

Tel.: +221.33.822.5919 - Fax: +221.33 .823 .5368$

E-mail: camaramakhtar@yahoo.fr

Key words: Respiratory tract infections; Haemophilus influenza; Moraxella catarrhalis; Streptococcus pneumonia; Antibiotic resistance.

Acknowledgements: the authors would thank all who have contributed to the success of this study.

Contributions: MC, AsD, CSBB, ON, DB, JBND, conceived and designed the experiments; $\mathrm{AbD}, \mathrm{AmD}, \mathrm{AF}$, analysed and interpreted the data; AsD, MC, wrote the first draft of the manuscript; AsD, MC, AmD, $\mathrm{MN}, \mathrm{ON}, \mathrm{CSBB}$, reviewed and approved of the final manuscript.

Funding: this work was partly supported by GlaxoSmithKline (GSK).

Conflict of interest: the authors declare no potential conflict of interst.

Received for publication: 17 December 2016.

Revision received: 14 February 2017.

Accepted for publication: 14 February 2017.

(C) Copyright M. Camara et al., 2017

Licensee PAGEPress, Italy

Microbiologia Medica 2017; 32:6489

doi:10.4081/mm.2017.6489

This article is distributed under the terms of the Creative Commons Attribution Noncommercial License (by-nc 4.0) which permits any noncommercial use, distribution, and reproduction in any medium, provided the original author(s) and source are credited. susceptibility to commonly used antibiotics tested by E-test.

Results. Among H. influenzae isolates, $10.5 \%$ were resistant to ampicillin (all $\beta$-lactamase-positive), and $88.9 \%$ were susceptible to cefaclor. High $\beta$-lactam resistance rates (penicillin: $31.3 \%$ and cephalosporins: 18.7 to $31.3 \%$ ) had been observed among S. pneumonia strains. Only $50 \%$ of isolates were susceptible to azithromycine. $91.3 \%$ of $M$. catarrhalis isolates $\beta$-lactamases producers were resistant to ampicillin while susceptible to the most tested antibiotics.

Conclusions. Except M. catarrhalis $\beta$-lactamases producing strains, frequency of antibiotic resistance was mainly observed among $S$. pneumoniae, and to a lesser extent among $H$. influenzae clinical isolates, suggesting the need for continuous surveillance of antimicrobial resistance patterns in the management of RTIs.

\section{Introduction}

Respiratory tract infections (RTIs) are the main cause of morbidity and mortality worldwide (7). Such diseases mainly involve bacteria such as Streptococcus pneumoniae, Haemophilus influenzae and Moraxella catarrhalis. In Africa, acute respiratory infections induce $16 \%$ of death in children under 5 years old (15). Despite this high burden, there are few studies reporting updates of antimicrobial susceptibility of respiratory pathogens in Africa (8). There are limited antimicrobial surveillance data in Africa, and results from one country may not be applicable to an another because of epidemiological discrepancies.

This makes surveillance of antibiotic resistance very important for guiding empirical therapy, especially in Africa, where health structures and patients must often rely on affordable first-line antibiotics that may have lost their clinical effectiveness (8).

Effective empirical therapy of bacterial diseases requires knowledge on local antimicrobial resistance (AMR) patterns since respiratory tract infection are usually treated without identification of the causal agent or its antibiotics susceptibility profile.

The objectives of this study were to identify these clin- 
ical isolates, and to assess their susceptibility to several antibiotics.

\section{Materials and methods}

\section{Sample collection}

We analysed data from paediatric samples collected in patient aged between 1 to 60 months visiting two medical centers (Abass NDAO teaching hospital and Roi BAUDOUIN hospital) in Dakar, Senegal.

From October 2014 to October 2015, sputum (21), bronchoalveolar lavage (19), acute otitis media effusions (01), blood (01), sinus fluids (11), and throat swab (05) samples were collected and referred to the biotechnology unit of the laboratory of bacteriology and virology of Aristide Le Dantec university teaching hospital for routine bacterial tests.

The samples were immediately cultured and the strains isolated were then identified according to the standard methods of microbiology. H. influenzae, S. pneumoniae, and $M$. catarrhalis strains were identified if the bacterial load was at least $10^{5} \mathrm{CFU} / \mathrm{mL}$.

\section{Identification of bacterial isolates}

$H$. influenzae was identified by its macroscopic aspects in culture (such as the growth of tiny, moist, and smooth grey colonies) as well as metabolic characteristics: absence of haemolysis, positive catalase and oxidase tests, growth in simultaneous presence of $\mathrm{X}$ and $\mathrm{V}$ factors, satellite growth around streaks of Staphylococcus aureus, and other biochemical features using API NH ${ }^{\circledR}$ galleria (BioMerieux, Marcyl'Étoile, France).

M. catarrhalis was identified by the presence of tiny, round, and smooth colonies; absence of haemolysis; positive catalase and oxidase tests; and others biochemical characters using $\mathrm{API}^{\circledR} \mathrm{NH}$ galleria (BioMerieux, France).

S. pneumoniae was identified by the presence of tiny, round, flat, and transparent colonies with central depression (checker piece and nail head colonies); haemolysis of $\alpha$-viridans; negative catalase and oxidase tests; absence of bileesculin hydrolysis; lysis by bile-salts; susceptibility to optochin; and other biochemical characters using API $^{\circledR}$ Strep (BioMerieux, France).

\section{Antibiotic susceptibility testing}

E-test method was used to study the antibiotic susceptibility of the three pathogens. Bacterial suspensions of each of the three pathogens were diluted to obtain a final concentration of $10^{5} \mathrm{CFU} / \mathrm{mL}$ (an optical density of 0.5 on the McFarland scale) and inoculated on Haemophilus Test Medium for $H$. influenzae, on chocolate agar supplemented with Polyvitex ${ }^{\circledR}$ for $M$. catarrhalis, and on Mueller-Hinton supplemented with 5\% sheep blood for S. pneumonia.

Strips (E-test, bioMerieux SA, LYON) containing selected antibiotics were then placed on the inoculated plates. Plates were then incubated at $37^{\circ} \mathrm{C}$ under $\mathrm{CO}_{2}$ atmosphere for 18-24 hours. Quality control for antimicrobial suscepti- bility testing was performed using the ATCC 49247 strain of H. influenzae, ATCC 49619 strains of S. pneumoniae. All antibiotics displayed acceptable minimal inhibitory concentrations (MICs) values compared to the control strains.

MICs were calculated as $\mathrm{MIC}_{50}$ (MIC inducing inhibition of $50 \%$ of isolates) and $\mathrm{MIC}_{90}$ (MIC inducing inhibition of $90 \%$ of isolates). Percentages of susceptibilities were determined based on Clinical Laboratory Standards Institute (CLSI) break points (4).

\section{Beta-lactamase tests}

The production of beta-lactamase has been investigated for $H$. influenzae and $M$. catarrhalis isolates using a nitrocefin-based test (Cefinase, Becton Dickinson Microbiology Systems, and Cockeysville, MD, USA).

\section{Analysis of results}

WHONET software (version 6) was used to analyse results of the antibacterial susceptibility test. $\mathrm{MIC}_{50}$, and $\mathrm{MIC}_{90}$ were calculated.

\section{Results}

In this study, 58 isolates (19 of $H$. influenzae, $16 \mathrm{~S}$. pneumoniae and 23 of $M$. catarrhalis) have been identified and tested for antibiotic susceptibility.

\section{Antibiotic susceptibility testing of $H$. influenzae}

The results of susceptibility testing for $H$. influenzae isolates are summarized in Table 1.

All $H$. influenzae isolates were susceptible to amoxicillin/clavulanic acid $\left(\mathrm{MIC}_{90}=1 \mathrm{mg} / \mathrm{L}\right)$, cephalosporins (cefuroxim $\mathrm{MIC}_{90}=3 \mathrm{mg} / \mathrm{L}$, cefixim $\mathrm{MIC}_{90}=0.094 \mathrm{mg} / \mathrm{L}$ ), fluoroquinolons (ciprofloxacin: $\mathrm{MIC}_{90}=0.094 \mathrm{mg} / \mathrm{L}$; levofloxacin: $\mathrm{MIC}_{90}=0.023 \mathrm{mg} / \mathrm{L}$; ofloxaxcin: $\mathrm{MIC}_{90}=0.016$ $\mathrm{mg} / \mathrm{L})$, and azytrhomycin $\left(\mathrm{MIC}_{90}=2 \mathrm{mg} / \mathrm{L}\right)$. However, resistance patterns to ampicillin (10.5\%), cefaclor $(11.2 \%)$ and /sulfamethoxazole/trimethoprim (100\%) were observed. All ampicillin-resistant isolates produced $\beta$-lactamase.

\section{Antibiotic susceptibility testing of $S$. pneumoniae}

Table 2 shows an overview of antibiotic susceptibility rates of $S$. pneumoniae. High $\beta$-lactams resistance rates had been observed among $S$. pneumoniae strains, including penicillin $\mathrm{G}(31.3 \%)$, cephalosporins (18.7 to $31.3 \%)$. All isolates were resistant to sulfamethoxazole/trimethoprim (100\%). Only $50 \%$ of $S$. pneumoniae isolates were susceptible to azithromycine while all isolates were susceptible to fluoroquinolone antibiotics (levofloxacin $\mathrm{MIC}_{90}=1 \mathrm{mg} / \mathrm{L}$, ofloxacin $\left.\mathrm{MIC}_{90}=2 \mathrm{mg} / \mathrm{L}\right)$ and clindamycin $\left(\mathrm{MIC}_{90}=0.19 \mathrm{mg} / \mathrm{L}\right)$.

\section{Antibiotic susceptibility testing of $M$. catarrhalis}

All $M$. catarrhalis isolates were susceptible to amoxicillin/clavulanic acid $\left(\mathrm{MIC}_{90}=0.5 \mathrm{mg} / \mathrm{L}\right)$ and macrolides (azythromycin $\quad \mathrm{MIC}_{90}=0.75 \mathrm{mg} / \mathrm{L}, \quad$ clarithromycin $\left.\mathrm{MIC}_{90}=1.5 \mathrm{mg} / \mathrm{L}\right)$ (Table 3). Fluoroquinolones tested 
showed very good activity (100\% and $95.2 \%$ for levofloxacin and ciprofloxacin, respectively), with low $\mathrm{MIC}_{90}$ $(0.094 \mathrm{mg} / \mathrm{L})$. Among isolates tested, $95.7 \%$ were susceptible to cefuroxim and cefaclor. However, $91.3 \%$ of $M$. catarrhalis isolates positive for $\beta$-lactamase were resistant to ampicillin (data not shown).

\section{Discussion}

The results from this study indicate a low frequency $(10.5 \%)$ of $H$. influenzae beta-lactamase producing strains, all resistant to ampicillin. Our results are in agreement with

Table 1. Susceptibility of Haemophilus influenzae isolates.

\begin{tabular}{|c|c|c|c|c|c|}
\hline Antibiotics & R, \% & $\mathrm{S}, \%$ & $\mathrm{MIC}_{50}, \mathrm{mg} / \mathrm{L}$ & $\mathrm{MIC}_{90}, \mathrm{mg} / \mathrm{L}$ & Range, mg/L \\
\hline Ampicillin & 10.5 & 89.5 & 0.25 & 1 & $0.04-1.5$ \\
\hline Amoxicillin/clavulanic acid & 0 & 100 & 0.5 & 1.5 & $0.125-2$ \\
\hline Cefuroxim & 0 & 100 & 1 & 3 & $0.19-4$ \\
\hline Cefaclor & 11.2 & 88.9 & 3 & 16 & $1.5-48$ \\
\hline Cefixim & 0 & 100 & 0.047 & 0.094 & $0.016-0.38$ \\
\hline Ciprofloxacin & 0 & 100 & 0.016 & 0.023 & $0.003-0.023$ \\
\hline Levofloxacin & 0 & 100 & 0.012 & 0.016 & $0.006-0.016$ \\
\hline Ofloxacin & 0 & 100 & 0.032 & 0.047 & $0.016-0.047$ \\
\hline Sulfamethoxazole/trimethoprim & 100 & 0 & 32 & 32 & $0.75-32$ \\
\hline Azithromycin & 0 & 100 & 1 & 2 & $0.38-2$ \\
\hline Clarithromycin & 5.3 & 94.7 & 4 & 8 & $1.5-12$ \\
\hline
\end{tabular}

R, Resistant; S, Susceptible; MIC, Minimum Inhibitory Concentration.

Table 2. Susceptibility of Streptococcus pneumonia isolates.

\begin{tabular}{|c|c|c|c|c|c|}
\hline Antibiotics & $\mathbf{R}, \%$ & $\mathrm{~S}, \%$ & $\mathrm{MIC}_{50}, \mathrm{mg} / \mathrm{L}$ & $\mathrm{MIC}_{90}, \mathrm{mg} / \mathrm{L}$ & Range, mg/L \\
\hline Penicillin G & 31.3 & 68.7 & 0.032 & 0.125 & $0.006-0.5$ \\
\hline Amoxicillin/clavulanic acid & 12.5 & 87.5 & 0.032 & 0.032 & $0.016-1$ \\
\hline Cefuroxim & 18.7 & 81.3 & 0.047 & 0.38 & $0.016-1.5$ \\
\hline Cefaclor & 31.3 & 68.7 & 0.75 & 1.5 & $0.094-2$ \\
\hline Cefixim & 24.5 & 75.5 & 0.38 & 1.5 & $0.125-1.5$ \\
\hline Levofloxacin & 0 & 100 & 0.5 & 1 & $0.38-1$ \\
\hline Ofloxacin & 0 & 100 & 1.5 & 2 & $1-2$ \\
\hline Sulfamethoxazole/trimethoprim & 100 & 0 & 3 & 32 & $0.064-32$ \\
\hline Clindamycin & 0 & 100 & 0.094 & 0.19 & $0.016-0.25$ \\
\hline Azithromycin & 50 & 50 & 0.5 & 1.5 & $0.016-1.5$ \\
\hline Clarithromycin & 19.7 & 81.3 & 0.064 & 0.125 & $0.016-0.125$ \\
\hline
\end{tabular}

R, Resistant; S, Susceptible; MIC, Minimum Inhibitory Concentration.

Table 3. Susceptibility of Moraxella catarrhalis isolates.

\begin{tabular}{|c|c|c|c|c|c|}
\hline Antibiotics & $\mathrm{R}, \%$ & S, $\%$ & $\mathrm{MIC}_{50}, \mathrm{mg} / \mathrm{L}$ & $\mathrm{MIC}_{90}, \mathrm{mg} / \mathrm{L}$ & Range, mg/L \\
\hline Amoxicillin/clavulanic acid & 0 & 100 & 0.064 & 0.5 & $0.016-3$ \\
\hline Cefuroxim & 4.3 & 95.7 & 0.75 & 2 & $0.064-12$ \\
\hline Cefaclor & 4.3 & 95.7 & 1.5 & 2 & $0.38-12$ \\
\hline Cefixim & 0 & 100 & 0.125 & 0.5 & $0.047-0.5$ \\
\hline Ciprofloxacin & 4.8 & 95.2 & 0.032 & 0.094 & $0.012-0.25$ \\
\hline Levofloxacin & 0 & 100 & 0.032 & 0.094 & $0.012-0.25$ \\
\hline Sulfamethoxazole/trimethoprim & 0 & 100 & 0.25 & 0.25 & $0.25-0.25$ \\
\hline Azithromycin & 0 & 100 & 0.064 & 0.75 & $0.023-0.75$ \\
\hline Clarithromycin & 0 & 100 & 0.047 & 1.5 & $0.023-1.5$ \\
\hline
\end{tabular}

R, Resistant; S, Susceptible; MIC, Minimum Inhibitory Concentration. 
findings from a previous study conducted in Dakar (10) and in other countries such as Italia (2), Northen Taiwan (14), Cuba (13), and South Africa (5). This suggests that beta-lactamase production could be the major mechanism of antibiotic resistance for these organisms.

A high rate $(100 \%)$ of $H$. influenzae resistance to cotrimoxazole was observed, contrasting therefore with results reported by Gueye et al., in Dakar in 2009 (10).

The cephalosporins, fluoroquinolones, and macrolides antimicrobial families were active and could therefore be used as alternative options in RTI treatment.

Penicillin G resistance in $S$. pneumoniae infection was observed in Africa as well as in other countries $(1,6)$. In our study, high resistance rate $(31.3 \%)$ of $S$. pneumoniae to penicillin $\mathrm{G}$ was observed, which is similar to data previously reported in a study conducted by Gueye and al. in Dakar (10). However, our data are not consistent with results reported in Sub-Saharan Africa in which resistance level remained below $6 \%$ overall (12). This is similar to findings from West Africa, especially in Gambia where surveillance studies have been carried out extensively and showed that only $6.6 \%$ of the isolates had intermediate resistance and none had full resistance to penicillin (3). Resistance to sulfamethoxazole/trimethoprim were detected in all isolates tested. This is in disagreement with results from a study conducted in Dakar in 2009 (10). However, increasing resistance rates to sulfamethoxazole/trimethoprim have been reported between 2003 and 2006 in the East Africa region (9).

M. catarrhalis isolates showed high susceptibilities towards most of the antibiotics tested, except for $\beta$-lactamase positive strains for which higher rate of resistance (91.3\%) was observed. In our study, we observed that betalactamase production was the primary mechanism of ampicillin resistance for $M$. catarrhalis; all isolates that were resistant to ampicillin were beta-lactamase producer. Similar results were observed in a previous surveillance study conducted in other parts of the world (11).

\section{Conclusions}

In summary, the results from this study indicate that none of the isolates exhibited multiple resistances patterns. Except M. catarrhalis ampicillin-resistant $\beta$-lactamases producing strains, frequency of antibiotic resistance was mainly observed among $S$. pneumoniae, and to a lesser extent among $H$. influenzae clinical isolates. Overall, cephalosporins, fluoroquinolones, and macrolides remain active on these three pathogens and could be used as alternative treatment for RTIs in association with continuous surveillance of antimicrobial resistance patterns.

\section{References}

1. Benbachir M, Benredjed S, Boye CS, et al. Two-year surveillance of antibiotic resistance in S.pneumoniae in four African cities. Antimicrob Agents Chemother 2001;45:627-9.

2. Cardines R, Giufre M, Pompilio A, et al. Haemophilus influenzae in children with cystic fibrosis: antimicrobial susceptibility, molecular epidemiology, distribution of adhesins and biofilm formation. Int J Med Microbiol 2012;302:45-52.

3. Cheung YB, Zaman SM, Nsekpong ED, et al. Nasopharyngeal carriage of S. pneumoniae in Gambian children who participated in a 9-valent pneumococcal conjugate vaccine trial and in their younger siblings. Pediatr Infect Dis J 2009;28:990-5.

4. Clinical and Laboratory Standards Institute. M100-S16. Performance standards for antimicrobial disc susceptibility tests; Sixteenth edition. Approved Standard. Wayne. PA: CLSI. 2006.

5. Crowther-Gibson P, Govender N, Lewis DA, et al. Part IV. Human infections and antibiotic resistance. South Afr Med J 2011;101:567-78.

6. Granizo JJ, Aguilar L, Casal J, et al. S. pneumoniae resistance to erythromycin and penicillin in relation to macrolide and betalactam consumption in Spain (1979-1997). J Antimicrobial Chemother 2000;46:767-73.

7. Jacobs E, Dalhoff A, Korfmann G. Susceptibility patterns of bacterial isolates from hospitalised patients with respiratory tract infections (MOXIAKTIV Study). Int $\mathrm{J}$ Antimicrob Agents 2009;33:52-7.

8. Kariuki S, Dougan G. Antibacterial resistance in sub-Saharan Africa: an underestimated emergency. Ann NY Acad Sci 2014; 1323:43-55.

9. Mudhune S, Wamae M, Network Surveillance for Pneumococcal Disease in the East African Region. Report on invasive disease and meningitis due to $\mathrm{H}$. influenzae and $\mathrm{S}$. pneumoniae from the Network for Surveillance of Pneumococcal Disease in the East African Region. Clin Infect Dis 2009;48:S147-52.

10. Ndiaye AG, Boye CS, Hounkponou E, et al. Antimicrobial susceptibility of select respiratory tract pathogens in Dakar, Senegal. J Infect Dev Ctries 2009;3:660-6.

11. Sahm DF, Brown NP, Thornsberry C, et al. Antimicrobial susceptibility profiles among common respiratory tract pathogens: a global perspective. Postgrad Med 2008;120:16-23.

12. Samuel K, Gordon D. Antibacterial resistance in sub-Saharan Africa: an underestimated emergency. Ann NY Acad Sci 2014; 1323:43-55.

13. Tamargo I, Fuentes K, Llop A, et al. High levels of multiple antibiotic resistance among 938 Haemophilus influenzae type b meningitis isolates from Cuba (1990-2002). J Antimicrob Chemother 2003;52:695-8.

14. Wang SR, Lo WT, Chou CY, et al. Low rate of nasopharyngeal carriage and high rate of ampicillin resistance for Haemophilus influenzae among healthy children younger than 5 years old in northern Taiwan. J Microbiol Immunol Infect 2008;41:32-40.

15. World Health Organization. Word Health. Part III. Global health indicators. 2014. Available from: http://www.who.int/gho/publications/world_health_statistics/EN_WHS2014_Part3.pdf 